Mohammad Hayal Alotaibi, Bakr F. Abdel-Wahab, Obaid Fahad Aldosari, Amany S. Hegazy, Benson M. Kariuki* and Gamal A. El-Hiti*

\title{
The crystal structure of $\mathbf{N}$-(7-(4-fluorobenzylidene)-3-(4-fluorophenyl)-3, 3a,4,5,6,7-hexahydro- $2 \mathrm{H}$-indazole-2-carbonothioyl)benzamide, $\mathrm{C}_{28} \mathrm{H}_{23} \mathrm{~F}_{2} \mathrm{~N}_{3} \mathrm{OS}$
}

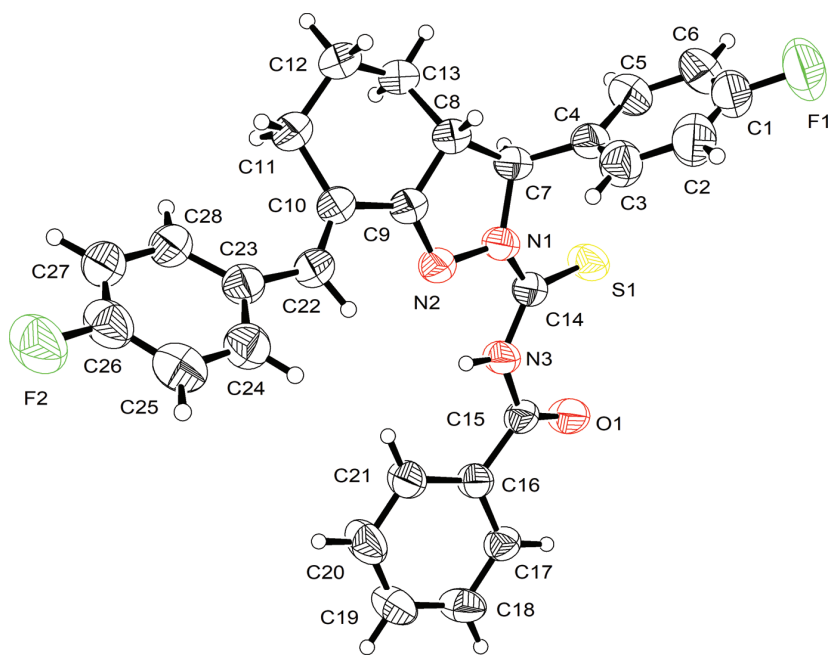

https://doi.org/10.1515/ncrs-2019-0281

Received April 17, 2019; accepted June 4, 2019; available online July 19, 2019

\section{Abstract}

$\mathrm{C}_{28} \mathrm{H}_{23} \mathrm{~F}_{2} \mathrm{~N}_{3} \mathrm{OS}$, monoclinic, $I 2 / a$ (no. 15), $a=20.3481(8) \AA$, $b=10.2647(4) \AA, \quad c=23.6975(11) \AA, \quad \beta=105.317(5)^{\circ}$,

*Corresponding authors: Benson M. Kariuki, School of Chemistry, Cardiff University, Main Building, Park Place, Cardiff CF10 3AT, UK, e-mail: kariukib@cardiff.ac.uk; and Gamal A. El-Hiti, Department of Optometry, College of Applied Medical Sciences, King Saud University, P.O. Box 10219, Riyadh 11433, Saudi Arabia, e-mail: gelhiti@ksu.edu.sa

Mohammad Hayal Alotaibi: National Center for Petrochemicals Technology, King Abdulaziz City for Science and Technology, P.O. Box 6086, Riyadh 11442, Saudi Arabia

Bakr F. Abdel-Wahab: Department of Chemistry, College of Science and Humanities, Shaqra University, Duwadimi, Saudi Arabia; and Applied Organic Chemistry Department, National Research Centre, Dokki, Giza, Egypt

Obaid Fahad Aldosari: Department of Chemistry, College of Science and Human Studies at Hautat Sudair, Majmaah University, P.O. Box 66, 11952 Majmaah, Saudi Arabia; and Chemistry Department, College of Science and Humanities, Prince Sattam bin Abdulaziz University, P.O. Box 83, 11942 Alkharj, Saudi Arabia

Amany S. Hegazy: School of Chemistry, Cardiff University, Main Building, Park Place, Cardiff CF10 3AT, UK
$V=4773.8(4) \AA^{3}, Z=8, R_{\mathrm{gt}}(F)=0.0489, w R_{\mathrm{ref}}\left(F^{2}\right)=0.1543$,

$T=296(2) \mathrm{K}$.

CCDC no.: 1920669

The crystal structure is shown in the figure. Table 1 contains crystallographic data and Table 2 contains the list of the atoms including atomic coordinates and displacement parameters.

Table 1: Data collection and handling.

\begin{tabular}{ll}
\hline Crystal: & Colourless block \\
Size: & $0.27 \times 0.26 \times 0.12 \mathrm{~mm}$ \\
Wavelength: & Mo $K \alpha$ radiation $(0.71073 \AA$ A $)$ \\
$\mu:$ & $0.18 \mathrm{~mm}^{-1}$ \\
Diffractometer, scan mode: & SuperNova, $\omega$-scans \\
$\theta_{\text {max }}$, completeness: & $29.7^{\circ},>93 \%$ (up to 25.2, $\left.>99 \%\right)$ \\
$N(h k l)_{\text {measured }}, N(h k l)_{\text {unique }}, R_{\text {int }}:$ & $39559,6270,0.032$ \\
Criterion for $I_{\text {obs }}, N(h k l)_{\text {gt }}:$ & $I_{\text {obs }}>2 \sigma\left(I_{\text {obs }}\right), 3722$ \\
$N(\text { param })_{\text {refined }}:$ & 264 \\
Programs: & CrysAlis \\
& Wing $[1]$, SHELX [2, 3], \\
&
\end{tabular}

\section{Source of materials}

The title compound was synthesized from reaction of an equimolar mixture of 7-(4-fluorobenzylidene)-3-(4fluorophenyl)-3,3a,4,5,6,7-hexahydro- $2 \mathrm{H}$-indazole and benzoyl isothiocyanate in anhydrous ethanol under reflux for $2 \mathrm{~h}$. The crude product was recrystallized from dimethylformamide to give colourless crystals in 82\% yield (Mp. $\left.246-248^{\circ} \mathrm{C}\right)$.

\section{Experimental details}

All hydrogen atoms were placed in calculated positions and refined using a riding model. The $\mathrm{N}-\mathrm{H}$ bond was fixed at $0.86 \AA$ (AFIX 43 instruction in SHELXL $[2,3]$ ), with displacement parameters set to 1.2 times $U_{\text {eq }}(\mathrm{N})$. C $-\mathrm{H}$ distances for $\mathrm{sp}^{2}$ carbon atoms were set to $0.93 \AA$ (AFIX 43) and $U_{\text {iso }}(\mathrm{H})$ set to 1.2 times $U_{\text {eq }}(\mathrm{C})$. The methine $\mathrm{C}-\mathrm{H}$ distance was set to $0.98 \AA$ (AFIX 13) and $U_{\text {iso }}(\mathrm{H})$ set to 1.2 times $U_{\text {eq }}(\mathrm{C})$. The methylene $\mathrm{C}-\mathrm{H}$ distances were set to $0.97 \AA$ (AFIX 23) and $U_{\text {iso }}(\mathrm{H})$ set to 1.2 times $U_{\text {eq }}(\mathrm{C})$. The phenyl ring is disordered and was refined with restrained geometry to form regular hexagons and restrained displacement parameters 
Table 2: Fractional atomic coordinates and isotropic or equivalent isotropic displacement parameters $\left(\AA^{2}\right)$.

\begin{tabular}{|c|c|c|c|c|}
\hline Atom & $x$ & $y$ & $z$ & $U_{\text {iso }} * / U_{\text {eq }}$ \\
\hline $\mathrm{C} 1$ & $0.29286(13)$ & $-0.5248(3)$ & $0.24724(15)$ & $0.1024(9)$ \\
\hline $\mathrm{C} 2$ & $0.30439(13)$ & $-0.3979(3)$ & $0.25847(12)$ & $0.0997(8)$ \\
\hline $\mathrm{H} 2$ & 0.303263 & -0.362985 & 0.294425 & $120^{*}$ \\
\hline C3 & $.31807(12)$ & $-0.3193(3)$ & $0.21515(11)$ & $0.0848(6)$ \\
\hline H3 & 0.326086 & -0.230924 & 0.222449 & $0.102^{*}$ \\
\hline $\mathrm{C} 4$ & $0.32004(10)$ & $-0.3690(2)$ & $0.16194(10)$ & $0.0709(5)$ \\
\hline C5 & 30884(14) & $-0.5001(2)$ & 0.1524 & $66(8)$ \\
\hline H5 & 0.310596 & -0.536500 & 0.116986 & $0.116^{*}$ \\
\hline C6 & $0.29482(16)$ & $-0.5791(3)$ & $0.19591(17)$ & $0.1128(10)$ \\
\hline H6 & 0.286958 & -0.667787 & 0.189478 & $0.135^{\star}$ \\
\hline$C 7$ & $0.33851(10)$ & $0.28349(19)$ & $0.11630(9)$ & $0.0673(5)$ \\
\hline $\mathrm{H} 7$ & 0.334939 & -0.333695 & 0.080434 & $0.081^{*}$ \\
\hline $\mathrm{C} 8$ & $0.41031(9)$ & $-0.2236(2)$ & $0.13749(9)$ & $0.0687(5)$ \\
\hline H8 & 0.426561 & -0.237276 & 0.179833 & $0.082^{*}$ \\
\hline C9 & $0.39820(9)$ & $0.08113(19)$ & 0.12 & $6(5)$ \\
\hline 10 & $0.45453(9)$ & $19(2)$ & $5(9)$ & $2(5)$ \\
\hline 11 & $0.52028(10)$ & $-0.0441(2)$ & $0.12734(11)$ & $0.0796(6)$ \\
\hline $11 \mathrm{~A}$ & 0.558128 & 0.000816 & 0.153653 & $0.095^{*}$ \\
\hline $\mathrm{H} 11 \mathrm{~B}$ & 0.522371 & -0.025831 & 0.087694 & $0.095^{*}$ \\
\hline 12 & $0.52988(10)$ & $-0.1910(2)$ & $0.13815(11)$ & $.0801(6)$ \\
\hline $\mathrm{H} 12 \mathrm{~A}$ & 0.566937 & -0.221034 & 0.122779 & $0.096^{*}$ \\
\hline $\mathrm{H} 12 \mathrm{~B}$ & 0.542544 & -0 & 59 & $96 *$ \\
\hline C13 & $0.46581(10)$ & $-0.2688(2)$ & $0.10973(10)$ & $0.0767(6)$ \\
\hline $13 \mathrm{~A}$ & 0.45 & -0.253247 & 375 & $0.092 *$ \\
\hline $\mathrm{H} 13 \mathrm{~B}$ & 0.474220 & -0.361245 & 0.116397 & $0.092^{*}$ \\
\hline C14 & $0.22965(9)$ & $-0.16202(18)$ & (9) & (5) \\
\hline C15 & $0.14029(8)$ & $0.00507(18)$ & 0.03 & $0.0616(5)$ \\
\hline $\mathrm{C} 16^{\mathrm{a}}$ & $0.1329(3)$ & $0.1480(3)$ & $0.0181(3)$ & $0.061(2)$ \\
\hline $\mathrm{C} 17^{\mathrm{a}}$ & 1(2) & (6) & (4) & $0.064(2)$ \\
\hline $\mathrm{H} 17^{\mathrm{a}}$ & 0.030541 & 0.143861 & 0.004 & $0.076^{*}$ \\
\hline $\mathrm{C} 18^{\mathrm{a}}$ & $.05606(16)$ & $0.3294(6)$ & $-0.0090(3)$ & $0.081(4)$ \\
\hline $\mathrm{H} 18^{\mathrm{a}}$ & 0.012057 & 62997 & -0.018094 & $0.097^{*}$ \\
\hline $\mathrm{C} 19^{\mathrm{a}}$ & $0.1107(2)$ & $0.4106(4)$ & $-0.0090(2)$ & $0.0849(16)$ \\
\hline $\mathrm{H} 19^{\mathrm{a}}$ & 0.103339 & 0.498396 & -0.018125 & $0.102^{*}$ \\
\hline $\mathrm{C} 20^{\mathrm{a}}$ & $.17651(17)$ & $0.3604(3)$ & $0.0045(2)$ & $0.0951(17)$ \\
\hline $\mathrm{H} 2 \mathrm{O}^{\mathrm{a}}$ & 0.213106 & 0.414661 & 0.004487 & $0.114^{*}$ \\
\hline $\mathrm{C} 21^{\mathrm{a}}$ & 1875 & (3) & (2) & $0.0785(14)$ \\
\hline $\mathrm{H} 21^{\mathrm{a}}$ & 0.231592 & 0.195525 & 0.027129 & $0.094^{*}$ \\
\hline $\mathrm{C} 16 \mathrm{~A}^{\mathrm{b}}$ & 0.1 & $489(5)$ & $.0294(4)$ & 0619(19) \\
\hline$C 17 A^{b}$ & $0.0700(3)$ & $0.1954(8)$ & $-0.0060(5)$ & $0.067(2)$ \\
\hline $\mathrm{H} 17 \mathrm{~A}^{\mathrm{b}}$ & 0.038356 & 0.137217 & -0.027968 & 0.080 * \\
\hline $\mathrm{C} 18 \mathrm{~A}^{\mathrm{b}}$ & $0.0557(3)$ & $0.3266(8)$ & $-0.0090(4)$ & $0.081(4)$ \\
\hline $\mathrm{H} 18 \mathrm{~A}^{\mathrm{b}}$ & 0.014729 & 0.356630 & -0.033024 & $0.097^{*}$ \\
\hline C19A $A^{b}$ & $0.1018(3)$ & $0.4130(5)$ & $0.0235(3)$ & $0.0840(17)$ \\
\hline$H 19 A^{b}$ & 0.091479 & 0.501391 & 0.022123 & $0.101^{*}$ \\
\hline$C 20 A^{b}$ & $0.1631(2)$ & $0.3696(4)$ & $0.0580(3)$ & $0.0930(19)$ \\
\hline $\mathrm{H} 20 \mathrm{~A}^{\mathrm{b}}$ & 0.194948 & 0.428809 & 0.078834 & $0.112^{\star}$ \\
\hline$C 21 A^{b}$ & $0.1774(2)$ & $0.2374(4)$ & $0.0616(3)$ & $0.0762(15)$ \\
\hline$H 21 A^{b}$ & 0.218433 & 0.208039 & 0.085959 & $0.091^{*}$ \\
\hline $\mathrm{C} 22$ & $0.44478(10)$ & $0.1351(2)$ & $0.15006(9)$ & $0.0687(5)$ \\
\hline $\mathrm{H} 22$ & 0.400694 & 0.153165 & 0.152123 & $0.082^{*}$ \\
\hline $\mathrm{C} 23$ & $0.49145(10)$ & $0.2462(2)$ & $0.16333(8)$ & $0.0669(5)$ \\
\hline 24 & $0.47536(12)$ & $0.3456(2)$ & $0.19732(9)$ & $0.0787(6)$ \\
\hline $\mathrm{H} 24$ & 0.435538 & 0.339254 & 0.209383 & $0.094^{*}$ \\
\hline $\mathrm{C} 25$ & $0.51670(14)$ & $0.4534(2)$ & $0.21371(11)$ & $0.0895(7)$ \\
\hline
\end{tabular}

Table 2 (continued)

\begin{tabular}{lrrrr}
\hline Atom & $\boldsymbol{x}$ & $\boldsymbol{y}$ & $\boldsymbol{Z}$ & $\boldsymbol{U}_{\text {iso }} \boldsymbol{U}_{\text {eq }}$ \\
\hline H25 & 0.505255 & 0.518707 & 0.236592 & $0.107^{*}$ \\
C26 & $0.57466(13)$ & $0.4613(2)$ & $0.19542(11)$ & $0.0864(7)$ \\
C27 & $0.59160(11)$ & $0.3698(2)$ & $0.16056(11)$ & $0.0842(6)$ \\
H27 & 0.630873 & 0.379194 & 0.147888 & $0.101^{*}$ \\
C28 & $0.55015(10)$ & $0.2624(2)$ & $0.14399(10)$ & $0.0762(6)$ \\
H28 & 0.561429 & 0.200044 & 0.119646 & $0.091^{*}$ \\
N1 & $0.29616(7)$ & $-0.16351(15)$ & $0.10253(7)$ & $0.0657(4)$ \\
N2 & $0.33473(7)$ & $-0.04890(15)$ & $0.10742(7)$ & $0.0660(4)$ \\
N3 & $0.20597(7)$ & $-0.03631(15)$ & $0.05803(7)$ & $0.0664(4)$ \\
H3A & 0.236086 & 0.024224 & 0.067574 & $0.080^{*}$ \\
O1 & $0.09265(7)$ & $-0.06788(14)$ & $0.01683(8)$ & $0.0878(5)$ \\
S1 & $0.18611(3)$ & $-0.29703(5)$ & $0.05185(3)$ & $0.0797(2)$ \\
F1 & $0.27951(10)$ & $-0.6031(2)$ & $0.28933(9)$ & $0.1500(8)$ \\
F2 & $0.61599(8)$ & $0.56611(15)$ & $0.21233(8)$ & $0.1208(6)$ \\
\hline
\end{tabular}

Occupancies: ${ }^{\mathrm{a}}=0.521(5),{ }^{\mathrm{b}}=0479(5)$.

(DFIX,SIMU) to give final occupancies of 0.479(5) and 0.521(5) for the two components.

\section{Discussion}

Many pyrazole derivatives have been synthesized and found to display antimicrobial, antiviral, anti-inflammatory, insecticidal and herbicidal activities [5-8]. The structure of the title compound has been obtained as part of a study of these materials [9].

The asymmetric unit consists of one molecule of the title compound (see the figure). The molecule consists of two fluorophenyl groups [A (F1, C1-C6) and D (F2, C23C28)], a hexahydroindazolyl group [B (N1, N2, C7-C13)] and a phenyl group [C (C16-C21)]. Ring $\mathbf{C}$ is disordered by a rotation of $35.3(3)^{\circ}$ about the $\mathrm{C} 15-\mathrm{C} 16$ bond. The cyclohexane segment (C8-C13) of $\mathbf{B}$ assumes a chair conformation. The pyrazolyl ring of $\mathbf{B}$ is planar with a maximum deviation of 0.037 (3) $\AA$ from the least squares plane of the ring. Related structures include cis-1-benzoyl-2-chloroacetyl4,5-hexamethylenepyrazolidine [10] and 2-benzyl 3-ethyl 6a-methyl 1-(benzoylcarbamothioyl)hexahydrocyclopenta[c] pyrazole-2,3,6a(1H)-tricarboxylate [11] in which the pyrazolyl rings are in envelope conformation. An intramolecular $\mathrm{N}-$ $\mathrm{H} \cdots \mathrm{N}$ contact is observed (N3 $\cdots \mathrm{N} 2$ distance of $2.575(2) \AA$; $\mathrm{N} 3-\mathrm{H} 3 \mathrm{~A} \cdots \mathrm{N} 2$ angle of $112.8^{\circ}$ ). Two molecules related by inversion symmetry are linked by weak non-classical C$\mathrm{H}$. . O interactions (C17 . . O 1 distance of 3.427(3) Å and C17$\mathrm{H} 17 \cdots \mathrm{O} 1$ angle of $158.4^{\circ}$ ) to form $\mathrm{R}^{2}{ }_{2}(10)$ rings. Comparably weak $\mathrm{C}-\mathrm{H} \cdots \mathrm{S}$ interactions also occur in the structure with $\mathrm{C} 27 \cdots \mathrm{S} 1$ distances of 3.676(3) A and C27-H27 .. S1 angles of $139.7^{\circ}$.

Acknowledgements: The authors would like to thank the Deanship of Scientific Research at Majmaah University for 
supporting this work under Project Number No. 1440-127, and to Cardiff University for continued support.

\section{References}

1. Rigaku Oxford Diffraction. CrysAlis PRO. Rigaku Oxford Diffraction, Yarnton, England (2015).

2. Sheldrick, G. M.: A short history of SHELX. Acta Crystallogr. A64 (2008) 112-122.

3. Sheldrick, G. M.: Crystal structure refinement with SHELXL. Acta Cryst. C71 (2015) 3-8.

4. Farrugia, L. J.: WinGX and ORTEP for Windows: an update. J. Appl. Crystallogr. 45 (2012) 849-854.

5. Naim, M. J.; Alam, O.; Nawaz, F.; Alam, M. J.; Alam, P.: Current status of pyrazole and its biological activities. J. Pharm. Bioallied Sci. 8 (2016) 2-17.

6. Sayed, G. H.; Azab, M. E.; Anwer, K. E.; Abdel Raouf, M.; Negm, N. A.: Pyrazole, pyrazolone and enaminonitrile pyrazole derivatives: Synthesis, characterization and potential in corrosion inhibition and antimicrobial applications. J. Mol. Liq. 252 (2018) 329-338.
7. Sidduri, A.; Budd, D. C.; Fuentes, M. E.; Lambros, T.; Ren, Y.; Roongta, V.; Schoenfeld, R. C.; Gillespie, P.; Stevenson, C. S.; Truitt, T.; Qian, Y.: Discovery of novel non-carboxylic acid 5amino-4-cyanopyrazole derivatives as potent and highly selective LPA1R antagonists. Bioorg. Med. Chem. Lett. 24 (2014) 4450-4454.

8. Faria, J. V.; Vegi, P. F.; Miguita, A. G. C.; Dos Santos, M. S.; Boechat, N.; Bernardino, A. M. R.: Recently reported biological activities of pyrazole compounds. Bioorg. Med. Chem. 25 (2017) 5891-5903.

9. Alotaibi, M. H.; Hanan, M. A.; Bakr, F. A.-W.; Amany, H. S.; Kariuki, B. M.; El-Hiti, G. A.: Crystal structure of 5-(5-(4chlorophenyl)-1-phenyl-1H-pyrazol-3-yl)-N-phenyl-2-amine, $\mathrm{C}_{23} \mathrm{H}_{16} \mathrm{ClN}_{5}$ O. Z. Kristallogr. NCS 234 (2019) 543-545.

10. Kapor, A.; Rakic, S.; Stancic, M.; Simon, L.; Talpas, G. S.; Bernath, G.; Engel, P.: Structure and conformation of cisbenzoyl-2-chloroacetyl-4,5-hexamethylenepyrazolidine: an x-ray study. J. Mol. Struct. 380 (1996) 85-91.

11. Gergely, J.; Morgan, J. B.; Overman, L. E.: Stereocontrolled synthesis of functionalized cis-cyclopentapyrazolidines by 1,3-dipolar cycloaddition reactions of azomethine imines. J. Org. Chem. 71 (2006) 9144-9152. 